\title{
Fegninto
}

Revista Legado de Arquitectura y Diseño

ISSN: 2007-3615

ISSN: 2448-749X

legado_fad@yahoo.com.mx

Universidad Autónoma del Estado de México

México

\section{CONCRECIÓN URBANA- ARQUITECTÓNICA DE LOS ASENTAMIENTOS DERIVADOS DE LA COLONIZACIÓN DEL SIGLO XIX EN MÉXICO}

Aguilar-Méndez, Carlos; Arista-González, Gerardo

CONCRECIÓN URBANA-ARQUITECTÓNICA DE LOS ASENTAMIENTOS DERIVADOS DE LA

COLONIZACION DEL SIGLO XIX EN MEXICO

Revista Legado de Arquitectura y Diseño, vol. 2019, núm. 26, 2019

Universidad Autónoma del Estado de México, México

Disponible en: http://www.redalyc.org/articulo.oa?id=477961406010

Esta obra está bajo una Licencia Creative Commons Atribución-NoComercial-SinDerivar 4.0 Internacional. 


\title{
CONCRECIÓN URBANA- ARQUITECTÓNICA DE LOS ASENTAMIENTOS DERIVADOS DE LA COLONIZACIÓN DEL SIGLO XIX EN MÉXICO
}

\author{
URBAN-ARCHITECTURAL CONCRETION OF \\ SETTLEMENTS DERIVED FROM THE 19TH \\ CENTURY COLONIZATION IN MEXICO
}

Carlos Aguilar-Méndez aguilar.carlos@uabc.edu.mx Universidad Autónoma de San Luis Potosí, México

Gerardo Arista-González garista@fh.uaslp.mx Universidad Autónoma de San Luis Potosí, México Revista Legado de Arquitectura y Diseño,
vol. 2019, núm. 26, 2019

Universidad Autónoma del Estado de México, México

Recepción: 03 Marzo 2019 Aprobación: 01 Junio 2019

Redalyc: http://www.redalyc.org/ articulo.oa?id=477961406010
Resumen: El siglo XIX en México se caracterizó por el poblamiento del territorio, con énfasis en la parte norte, derivado de una política colonizadora ejecutada por extranjeros. El objetivo de este trabajo es explicar el surgimiento y los grados de consolidación que presentaron los asentamientos poblacionales derivados y coadyuvar en el entendimiento de su trascendencia.

La metodología aborda el periodo de 1880 a 1910 con base en la aplicación de la política colonizadora. Se comienza con el análisis de la concreción espacial de la legislación y la identificación de las colonias emanadas. Posteriormente se muestra la caracterización de cuatro casos de estudio y un análisis comparativo de su medio físico, los habitantes, sus características urbanas y arquitectónicas.

Como resultado se vislumbró que la política colonizadora fue basada en especulaciones al firmarse 201 contratos y concretarse sólo 44 colonias. Los proyectos resultantes tomaron como base el modelo urbano estadounidense con cánones tendientes a la funcionalidad y racionalidad del espacio. La arquitectura introducida fue adecuada con base en los materiales y las características climáticas de la región. El aspecto social se derivó de las intenciones de los desarrolladores, los habitantes y el gobierno mexicano.

Se concluye que las características físicas de los proyectos coadyuvaron en el desarrollo de las poblaciones y la previsión de su futuro crecimiento a costa de una homogenización; la dimensión social, caracterizada por la discordancia de las intenciones de los actores, influyó en la apropiación del espacio y su crecimiento urbano y demográfico derivando en distintos grados de consolidación.

Palabras clave: colonización, concreción espacial, México, siglo XIX, trascendencia. Abstract: The XIX century in Mexico was characterized by the territorial occupation, mainly at north, derived from a colonizing policy executed by foreigners. The objective of this work is to explain the emergence and the different consolidation levels presented by the settlements derived from this policy and contribute to the knowledge of their transcendence.

The methodology analyze the period from 1880 to 1910 based on the colonization policy application. This work begins with an analysis of the legislation about his spatial concretion and the identification of the derived colonies. Subsequently is shown the characterization of four case studies and a comparative analysis of their physical environment, the inhabitants and their urban and architectural characteristics.

In the results is feasible to see the fact that the colonization policy was based on land speculation with 201 contracts signed and just 44 colonies materialized. The resulting projects were based on the American urban model with canons of functionality and rationality of the space. The architecture introduced was adequate in relation with the 
regional climatic characteristics and materials. The social aspect was derived from the intentions of the developers, the inhabitants and the Mexican government.

In conclusion, the physical characteristics of these projects contributed to their population's development and anticipated their future growth at the cost of a homogenization; the social dimension, characterized by the disagreement in the intentions of the actors, influenced the spatial appropriation and its urban and demographic growth resulting in different levels of consolidation.

Keywords: spatial concretion, Mexico, XIX century, transcendence, colonization .

\section{INTRODUCCIÓN}

El objeto de estudio del presente trabajo refiere a la concreción y trascendencia espacial de los asentamientos poblacionales, establecidos y habitados por extranjeros, resultantes de la implantación de proyectos urbano-arquitectónicos que derivaron de la política colonizadora decimonónica en México. Las Unidades de análisis refieren a los proyectos desarrollados en el periodo de 1880 y 1910, lapso en el que se concretó espacialmente la política colonizadora. Se abordan específicamente como casos de estudio los asentamientos de Ensenada en Baja California, Topolobampo y Los Mochis en Sinaloa y Diez Gutiérrez en San Luis Potosí, como muestra representativa de la diversidad de vertientes que tomó el fenómeno de estudio en su aplicación.

La estrategia de desarrollo colonizadora de México a finales del siglo XIX es una temática que ha sido abordada de manera principal desde el ámbito político y jurídico con resultados cuantitativos de corte agrario. Destaca el trabajo de Macías (2015), quien menciona del concepto de propiedad, su evolución y signi ficado en la sociedad mexicana, enmarcando un proyecto gubernamental fundamentado en la ideología liberal impuesta sobre la realidad social. DeVos ( 1995 ) proporciona una perspectiva del contexto histórico que derivó en el fenómeno de estudio ; el autor muestra una descripción de los acontecimientos integradores del periodo de colonización, de las instituciones involucradas, con énfasis en las intenciones del gobierno mexicano, así como los mecanismos para incentivar a la inversión extranjera.

\section{ES IMPORTANTE VISUALIZAR EL FENÓMENO DE MANERA HOLÍSTICA. relacionar y comparar los precedentes, características contextuales y nivel de concreción de los diferentes casos de implantación QUE SE DIERON DENTRO DEL FENÓMENO COLONIZADOR DECIMONÓNICO.}

Son escasos los trabajos referentes al análisis urbano arquitectónico, así como la trascendencia a nivel espacial del fenómeno colonizador del siglo XIX en el país. Existen estudios, principalmente históricos , que consideran la fundación de los proyectos de manera aislada; sin embargo, es importante visualizar el fenómeno de manera holística, relacionar y comparar los precedente, características contextuales y nivel de concreción de los diferentes casos de impltación que se dieron dentro del fenómeno colonizador decimonónico, mismos que llevaron a un distinto nivel de trascendencia en proyectos que provinieron de la misma política. 
Al respecto, Martínez (2009) aborda el fenómeno de la colonización desde el ámbito internacional y describe al contexto del fenómeno de colonización durante el siglo XIX en América Latina. GonzálezMilea (2016) presenta un referente urbano-arquitectónico del tema al considerar los valores del patrimonio moderno con el análisis de la fundación de ciudades en el siglo XXI desde la perspectiva de las colonias civiles y militares derivadas en el Norte de México. A nivel urbano arquitectónico uno de los principales trabajos que marca la pauta con relación al análisis espacial de corte longitudinal refiere a Calderón (2001), autora que presenta y analiza las características espaciales que precedieron el fenómeno colonizador y las implantadas posteriormente por las compañías deslindadoras en el caso de Ensenada, Baja California.

Se encuentra que existe un nicho de investigación en el abordaje del fenómeno desde su manifestación espacial con el análisis de las corrientes de pensamiento, las características contextuales a nivel global, nacional y local, y su resultado a nivel urbano arquitectónico. El problema de investigación que se aborda refiere al entendimiento de los desarrollos poblacionales como la materialización en el espacio de la estrategia de desarrollo colonizadora ; cómo surgen, cómo se materializan a nivel urbano arquitectónico y a qué obedecen sus características con la intención de entender cuál fue su trascendencia en el ámbito territorial, el proceso que se obtuvo y a qué se llegó.

El objetivo general del proyecto refiere a explicar el surgimiento y la trascendencia de los asentamientos poblacionales derivados de la implantación de proyectos urbano-arquitectónicos como resultado de la política colonizadora decimonónica en México. Se pretende contestar a las preguntas ¿Cómo es que los factores filosóficos, geográficos, políticos, económicos y socioculturales materializados en una estrategia colonizadora dieron lugar al surgimiento de asentamientos poblacionales en México entre 1880 y 1909?, ¿Cuál fue la incidencia de los factores físicos y culturales dentro del marco de la concreción en el espacio de los proyectos urbano-arquitectónicos resultantes? Y ¿Cuál fue la trascendencia de los proyectos resultantes dentro del ámbito urbano territorial?

Es por ello que en los resultados se presenta en primera instancia el contexto ideológico y sociocultural que precedió la colonización decimonónica en el país; se aborda la concreción espacial de la legislación colonizadora e identificación de las colonias derivadas; posteriormente se retoma el aspecto espacial del tema con el análisis de cuatro casos de estudio, y por último se presentan patrones y particularidades de la concreción y trascendencia urbano -arquitectónica de los proyectos derivados de la colonización del siglo XIX en México. 


\section{METODOLOGÍA}

La metodología es de corte longitudinal y abarca el periodo de 1880 a 1910; lapso establecido con base en la aplicación y concreción espacial de la política colonizadora en el país. La legislación referente al fenómeno colonizador inició desde 1824 con una invitación a extranjeros a establecerse en el país a cambio de terrenos nacionales; fue en 1882 que se concretó físicamente el primer proyecto de colonia derivado y en 1909 se abrogó la política colonizadora como precedente de la Revolución Mexicana (De-Vos, 1995).

Para lograr el objetivo general del trabajo se comenzó por definir los factores filosóficos, geográficos, políticos, económicos y socioculturales que dieron lugar al desarrollo de los proyectos urbano arquitectónicos en México a finales del siglo XIX. Posteriormente se recabó información referente al panorama nacional de la aplicación de la legislación colonizadora en el territorio . Los datos recopilados refieren a la ejecución de los proyectos: la identificación de las colonias consolidadas, su origen y la cuantificación de los resultados de la colonización.

Con la finalidad de presentar un análisis de la espacialidad de los proyectos derivados de la colonización decimonónica en México y la comparativa de sus patrones generales, particularidades y nivel de trascendencia se establecieron cuatro casos de estudio, con base en criterios de selección derivados del análisis de la política, su origen y aplicación. Los criterios de selección refieren a cumplir con una fecha de fundación dentro del periodo de estudio, presentar una ubicación representativa para el fenómeno colonizador a nivel nacional, representar diversidad en las características del proyecto referente a su origen, tipo de asentamiento y la diversidad de grados de consolidación.

Los casos de estudio seleccionados fueron las colonias de Ensenada en Baja California, Topolobampo y Los Mochis en Sinaloa y Diez Gutiérrez en San Luis Potosí. Su selección se justifica debido a que las cuatro colonias se establecieron en la década de 1880 , su ubicación es representativa al emplazarse en la región norte, dentro de los estados con mayor número de colonias establecidas durante el periodo establecido, tres desarrollos poblacionales refieren al norte como la zona con mayor número de proyectos concretados y el cuarto desarrollo representa el primer y mayor proyecto de colonización con corte agrario concretado en el país.
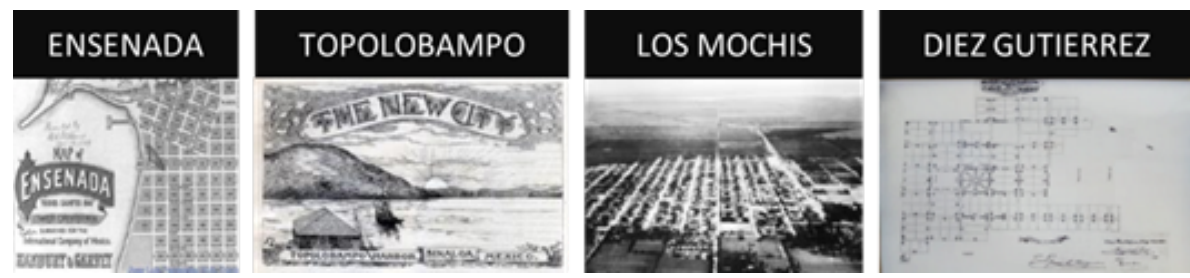

Figura 1. Unidades de análisis para el estudio de la espacialidad de los proyectos derivados de la colonización.

Fuente: Elaboración propia con base en los planos de Calderón (2001), Martínez (2009) y figuras de Lastras (1974). 
Con el objetivo de contrastar la concreción espacial de proyectos, sus patrones y particularidades, se tomó en cuenta el contexto urbano de cada caso de estudio con el análisis formal del modelo urbano, las influencias, la escala, la funcionalidad, los flujos, los elementos de organización, la traza, las calles, los lotes, las orientaciones, la distribución, la infraestructura y los servicios (Acuña Vigil, 2005). Con relación al análisis arquitectónico se analizan las categorías de análisis planteadas por Bruno Stagno (1997) y Norberg Schulz (2008): aspecto espacial, material, de adecuación al medio ambiente y las vivencias de la población.

Por último se conjuntaron los resultados del análisis comparativo de los casos de estudio, la caracterización a nivel urbano, arquitectónico y social, la caracterización contextual, los factores físicos y simbólicos específicos de los desarrollos poblacionales resultantes de la estrategia de desarrollo colonizadora y su influencia a posteriori para llegar al cumplimiento del objetivo general que refiere a explicar la concreción y la trascendencia de los asentamientos poblacionales derivados de la implantación de proyectos urbano-arquitectónicos como resultado de la política colonizadora de México en el siglo XIX.

\section{CONTEXTO IDEOLÓGICO Y SOCIOCULTURAL QUE PRECEDIO LA COLONIZACION DECIMONÓNICA EN MÉXICO}

El poblamiento del territorio mexicano a finales del siglo XIX, impulsado por medio de la estrategia de colonización, derivó de diversos factores contextuales de la época referentes a cuestiones intrínsecas yextrínsecas del territorio mexicano : corrientes de pensamiento como la ilustración y el liberalismo; acompañadas de acontecimientos históricos plasmados en las invasiones extranjeras y las guerrillas internas del país. Esta serie de factores derivaron en una crisis política manifestada en la confrontación entre liberales y conservadores, una crisis económica tendiente a la alza y una crisis agraria derivada de la deficiente distribución de la tierra (Martínez, 2009).

A lo largo del siglo XIX se impuso el liberalismo al consolidar fuerzas revolucionarias en Europa, así como al sentar las bases para las constituciones de los estados. En el sentido humanista del liberalismo, este refiere al principio fundamental de la dignidad de la persona, de la que se deriva un ámbito de autonomía o libertad individual para llevar a cabo sus tareas en la vida. En esta idea no le es permitido al Estado la intervención coercitiva en las materias que forman el ámbito de la autonomía individual. Como filosofía, el liberalismo se vio traducido al ámbito político y económico con resultados cuestionables en contraste con su pensamiento principal debido a su apego con el sistema capitalista (Alvarado, 1963). 


\section{El pensamiento liberal se plasmó en \\ LA VISIÓN DEL PORFIRIATO CON CARACTERÍSTICAS COMO \\ EL LIBRE MERCADO. LA ELIMINACIÓN DEL GASTO PÚBLICO POR LOS SERVICIOS SOCIALES, LA DESREGULACIÓN, LA PRIVATIZACIÓN Y LA ELIMINACIÓN DEL CONCEPTO DE BIEN PÚBLICO.}

El pensamiento liberal se plasmó en la visión del porfiriato con características como el libre mercado, la eliminación del gasto público por los servicios sociales, la desregulación, la privatización y la eliminación del concepto de bien público. Este fenómeno se tradujo en la implantación de colonias en emplazamientos estratégicos para el flujo del mercado. En el gobierno de Díaz se reconoció el problema nacional de corte agrario, que como afirma Macías (2015) responde a una mala distribución de la tierra y la crisis económica por la que pasaba el país. En 1883, el presidente Manuel González promulgó una ley colonizadora que dedicó un capítulo entero al tema del deslinde de los terrenos y otro al tema de las compañías, tanto deslindadoras como colonizadoras. Diversos empresarios, a partir de 1884, se presentaron en la Secretaría de Fomento con el objeto de celebrar con el Gobierno Federal contratos de colonización o de deslinde (Ledesma, 2003).

En la primera década, después de la promulgación de la ley, se fi rmaron más de 200 convenios, 145 de deslinde y 56 de colonización ; a pesar de esto durante el gobierno de Porfirio Díaz se establecieron sólo 60 colonias (De-Vos, 1995). El patrón que siguió su emplazamiento responde a nodos económicos importantes como los puntos mineros en el país, así como el seguimiento de la vía férrea existente y proyectada en el país; pautas que influyeron de manera latente en su espacialidad posterior a nivel urbano y arquitectónico. De las 60 colonias, 16 de ellas ofi ciales, 44 eran particulares y sólo 34 habitadas por extranjeros (figura 1).

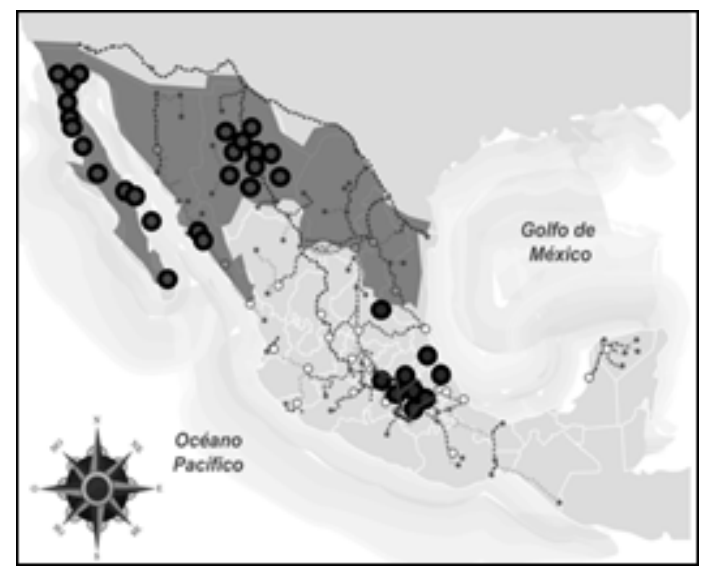

Figura 2. Localización de las 34 colonias particulares habitadas por extranjeros y contraste con la proyección de la vía del ferrocarril en el territorio mexicano. Fuente: Elaboración propia con base en De-Vos (1995). 


\section{ESPACIALIDAD DE CUATRO CASOS DE ESTUDIO : ENSENADA, TOPOLOBAMPO, LOS MOCHIS Y DIEZ GUTIÉRREZ}

Con la finalidad de presentar un panorama general de la espacialidad de los proyectos derivados de la colonización de México en el siglo XIX se presenta a continuación la caracterización , con base en datos de Calderón (2001), Lastras (1974 ) y Martínez (2009), de cuatro casos que representan la diversidad de factores que precedieron la materialización de los proyectos y los grados de concreción a los que se llegó . La primer dimensión que se aborda , para entender la gestación de los proyectos son sus antecedentes ; dada la cercanía y el interés expansionista y de explotación de los recursos naturales tres de los cuatro casos de estudio (Ensenada, Topolobampo y Los Mochis) re fieren a proyectos estadounidenses. El cuarto caso, Diez Gutiérrez, refiere al primer y más grande proyecto agrícola con procedencia italiana en el país. El área del asentamiento de Topolobampo , los Mochis y Diez Gutiérrez rondaba las 4000 y 5000 hectáreas , a diferencia de Ensenada que planteaba un proyecto sumamente ambicioso con un área mayor a las cinco millones de hectáreas.

Las familias que inmigraron al país buscaron una nueva alternativa de crecimiento económico, social y personal; mismo que encontraron en la propaganda sobre exaltada de las nuevas colonias derivadas de la estrategia colonizadora de México bajo el panorama de una tierra rica y factible de trabajar para establecer proyectos utópicos, comerciales o agrí́colas. Pese a eso un factor determinante en la consolidación y trascendencia de los proyectos refirió a la congruencia de objetivos y expectativas planteados por los inversionistas y el gobierno federal que lejos de visualizar el bienestar social priorizó el carácter económico y personal (Lastras, 1974).

Con relación al contexto geográfico y específicamente al emplazamiento de los proyectos, en Ensenada se tomó como factor principal la cercanía con la frontera norte y el acceso por la vía marítima, así como el clima templado, muy parecido al de California , con especulaciones de la vegetación y factibilidad del uso del suelo. En su gestación, la colonia comenzó con 371 viviendas y 1373 habitantes, quienes estaban habituados al modo de vida del suroeste de Estados Unidos con un clima y unas vistas sumamente parecidos (Calderón, 2001).

La selección del emplazamiento en Topolobampo tuvo una tónica similar, aunque sus inicios fueron sumamente distintos debido principalmente a la carencia de un proyecto que lo antecediera en el lugar. El puerto de Topolobampo está ubicado al noroeste del país sobre la costa oriental del Golfo de California, pertenece al municipio de Ahome, estado de Sinaloa y se encuentra a una distancia de $25 \mathrm{~km}$ de la Ciudad de Los Mochis, Sinaloa , y a $322 \mathrm{~km}$ de la entrada al Golfo de California y predomina el clima seco extremoso. Se seleccionó el espacio , pese a las características climáticas del emplazamiento , su topografia escarpada y la escasez de recursos hídricos, en la búsqueda de un puerto que pudiera ser 
la terminal de la futura ruta hacia el océano Pacífico, y se determinó que el punto más apropiado era Topolobampo.

Pese a los esfuerzos de su proyectista Owen las características adversas del emplazamiento y la incongruencia de aptitudes de los habitantes con relación a las labores que se requerían, el inicio del proyecto de Topolobampo tuvo circunstancias adversas que marcaron su carente concreción como colonia y la futura trascendencia en el ámbito territorial. De esta manera, los habitantes de Topolobampo se trasladarían 10 años después a un nuevo proyecto de ámbito capitalista que ofrecía una topografía apta para la agricultura y la capacidad de obtener el recurso hídrico que tanto careció al proyecto: Los Mochis (Lastras, 1974).

Con relación a Diez Gutiérrez, el primer y más grande proyecto agrario en el país, cabe destacar que su inicio fue marcado por una característica de crucial importancia: su gestación fue completamente contraria, si bien en los tres casos anteriores un proyecto poblacional atrajo a los futuros habitantes, en el caso de Diez Gutiérrez la llegada de los futuros colonos arrojó la necesidad de crear un proyecto urbano y arquitectónico que lo sustentara ; que sería llevado a cabo por los mismos habitantes . El poblado se instaló en primera instancia a los pobladores en San Antonio Guascamán, en el partido de cerritos, debido a la comisión monetaria que se podía obtener, pues era terrenos que no costarían nada al gobierno; así como de la previa expectativa del proyecto de una línea de ferrocarril que atravesaría el partido: la vía aguas calientes-Tampico.

Debido a la falta de comida, de techo y agua; el excesivo calor que afectaba a los italianos por ser originarios de tierras con climas fríos o templados, el desconocimiento de las costumbres del lugar y la alimentación ocasionaron en ellos enfermedades con consecuencias fatales por falta de médicos. Esto orilló a los jefes de familia a pedir el traslado de la colonia a la ciudad del maíz. En el segundo emplazamiento se tenía un clima templado; situado en la línea que divide la aridez esteparia del altiplano potosino y la tórrida serranía repleta de encinales y vegetación con ríos que bajan de la Sierra Madre Oriental (Martínez, 2009).

\section{CONCRECIÓN URBANA Y ARQUITECTÓNICA DE LOS PROYECTOS}

El caso de Ensenada, como aborda Calderón (2001), contó con un desarrollo previo que, si bien no marcó la pauta, proporcionó un sustrato para la implantación del proyecto colonizador. Esta población previa muestra reminiscencias del modelo hispanoamericano colonial con una plaza central que estructuró el espacio, rodeada por los dos elementos simbólicos de la población: la iglesia y el edificio de gobierno. Como resultado la lotificación se tornó con base en la plaza y posteriormente a las vialidades comerciales del proyecto. La plaza se ubica descentrada aunque en un sitio predominante dentro de la traza original. Las calles salen de cada una de las cuatro esquinas y enmarcan a la plaza.

No se enmarcan jerarquías sociales debido a que se trató de vender porciones de terreno para incrementar el beneficio económico, el 
conjunto urbano no constituyó un perímetro en cuadro y la orientación de esta traza previa obedeció al norte solar orientado a $45^{\circ}$ del norte geográfico. Posteriormente, el proyecto de la compañía internacional de México propuso un área diez veces mayor orientada hacia el norte magnético en la cual se relegó el carácter simbólico al desplazar la plaza de la ciudad y lotificar con base en las Townships con el objetivo de comercializar la mayor cantidad posible de terrenos (Calderón, 2001).

En el caso de Topolobampo, el modelo urbano tuvo una gran influencia, como menciona Lastras (1974) del modelo de Francia transportado a Washington. Sus flujos además de contar con trazos ortogonales adhirieron avenidas diagonales que cruzaban la traza propuesta. En este proyecto Owen describió minuciosamente los distintos ámbitos desde el trazado urbanístico y el sistema socioeconómico hasta las costumbres aceptadas y la rutina diaria. Aunado las avenidas fueron divididas por países con el objetivo de incidir en la identificación y socialización de las personas. La ubicación espacial dentro del lote fue central con el establecimiento de jardines en los lotes.

La ciudad quedaba dividida en cuatro sectores. Sus avenidas serían de 200 pies de ancho, calles de 100 pies y callejones de 50, todo a escuadra. Avenidas diagonales de gran amplitud para facilitar la circulación urbana. Las zonas residenciales, de comercio, fabril y marítima (con malecones, muelles, diques, canales y almacenes) determinados dentro del plano (Lastras, 1974).

La colonia Diez Gutiérrez retomó los caracteres simbólicos del modelo virreinal como resultado de la carencia de un proyecto urbanoarquitectónico que precediera el poblamiento. Como resultado del análisis del plano y las características mencionadas por Martínez (2009) se encontró que tomaron influencias de las poblaciones circundantes y agregaron la plaza y los dos órdenes simbólicos existentes en su trazado: la iglesia y el gobierno local. La traza de igual manera es reticular y se encuentra rotada a $45^{\circ}$ del norte magnético.

Con relación a la arquitectura legada por el fenómeno colonizador de México en el siglo XIX se muestran dos vertientes distintas. Cuando el proyecto fue previo a la llegada de los habitantes se tomaron referentes conocidos como en el caso de Ensenada y Los Mochis y se adecuaron al medio con los recursos materiales que se tenían en la región. En el caso de Ensenada se introdujo la técnica constructiva de marcos rígidos de madera denominada "Balloon Frame", misma que fue aceptada por su carácter económico, la facilidad de construcción sin necesidad de mano de obra experta, su fuerza estructural compatible con zonas sísmicas y la capacidad de apropiación al poderse construir en distintos estilos ( vernáculo americano, victoriano, búngalo). Las adecuaciones al clima re fieren a una ventilación cruzada por medio de ventanas de guillotina, con rejillas de ventilación entre la techumbre y el plafón, así como la suspensión del piso del terreno natural y la ventilación intermedia. La espacialidad de la arquitectura se caracterizó por situar las edificaciones al centro o al costado del lote con una división del espacio público y privado 
por medio de un elemento de transición denominado pórtico (Aguilar, 2015).

La segunda vertiente refiere a un proyecto posterior a la llegada de los habitantes, situación en la cual se tomaron como referencia las costumbres y materiales locales. Por este hecho en Topolobampo las primeras casas de ramas imitando a los pobladores nativos de la bahía se apegaron a las costumbres de los nativos, hecho que los ayudó a subsistir. Posteriormente en 1888, al igual que en Diez Gutiérrez erigieron nuevas casas con los materiales de la región: adobe y paja que se adecuaron al clima de la región (Martínez, 2009).

\section{TRASCENDENCIA DE LOS PROYECTOS}

De los cuatro casos de estudio dos perduraron y mostraron un crecimiento gradual ascendente a lo largo del tiempo: Ensenada y Los Mochis. Ensenada se fundó en 1882 y en 1886 se implantó la colonia Carlos Pacheco con un crecimiento gradual de su demografía y de la mancha urbana. En el caso de Topolobampo su fundación fue en 1903 y presentó las características mencionadas en la Colonia Carlos Pacheco en Ensenada. Estos dos proyectos presentan la característica de haber contado con un asentamiento previo que fungió como sustrato para empezar las construcciones arquitectónicas y el trazado urbano de la futura ciudad. A pesar de que estas colonias presentaron un crecimiento continuo su concreción tuvo que hacer frente a factores adversos tales como el incumplimiento de objetivos por parte de la compañía colonizadora, incongruencia entre la publicidad ofertada del proyecto y las características físicas del lugar y la carencia de simbolismos dentro del proyecto que propiciaran la identificación por parte del habitante.

Los proyectos colonizadores de Topolobampo y Diez Gutiérrez se vieron coartados por diversos factores. El proyecto de Topolobampo nació en 1886 con Albert Owen, pero debido a la carencia de un proyecto antecedente, el incumplimiento de objetivos por parte de la compañía, una incongruencia del modelo económico propuesto y principalmente carencias naturales del medio físico, incompatibilidad de los modos de vida e incongruencia de la oferta y la realidad, en 1897 se da por cesado el proyecto y renace en los Mochis por los mismos habitantes (Lastras, 1974).

Diez Gutiérrez, fundada en 1882 y trasladada en 1883, tuvo que hacer frente a los factores mencionados anteriormente, pero aunado a los conflictos bélicos de la Revolución Mexicana vio la desaparición de su proyecto en 1909. Treinta años después renació la colonia, por los mismos habitantes y continuada como un proyecto cerrado que se integra por los descendientes de las primeras familias (Martínez, 2009).

Espacialmente las colonias presentan una trascendencia regional a través de la pauta que marcó el modelo urbano introducido, los cánones y dimensiones del diseño que marcaron el crecimiento posterior de las ciudades. Aunado es factible de observar por medio de la introducción y pervivencia de la infraestructura y servicios dotados por los proyectos; en 
el caso de ensenada se tiene el legado de tres hoteles, la línea de teléfono, alumbrado púbico, líneas de vapores, medios de comunicación impresos y la red de Agua potable (Calderón, 2001). En el caso de Topolobampo se tiene además la construcción del primer sistema de riego, el cultivo de la tierra con sistemas modernos. De igual manera se vislumbra como resultado un crecimiento demográfico y urbano acelerado, de igual manera el comercio y la agricultura circundante y su constitución como cabecera municipal (Lastras, 1974). Diez Gutiérrez legó una trascendencia no física sino simbólica como el primer y más grande proyecto agrario en el país, donde actualmente viven 120 descendientes de los migrantes fundadores (Martínez, 2009).

A nivel arquitectónico su legado es factible de observar a través de la pervivencia constructiva de las edificaciones legadas por las compañías extranjeras, con un sistema constructivo introducido y adecuado a las características del medio constituido por marcos rígidos de madera denominado "balloon frame" y por la pervivencia de las características de diseño como el emplazamiento dentro de las construcciones al frente del lote y los elementos arquitectónicos tales como la ventana de bahía, el espacio porticado y los sistemas de ventilación cruzada por medio de rejillas (Aguilar, 2015).

\section{CONCLUSIONES Y RECOMENDACIONES}

La colonización del siglo XIX en México, derivada de una estrategia de desarrollo traducida como una política de poblamiento, dio como resultado la implantación de 34 colonias extranjeras, mismas que marcaron el desarrollo regional posterior en el norte de México por medio de características urbanas y arquitectónicas introducidas.

Los proyectos y ciudades derivadas tienen la característica de ser ciudades "relativamente jóvenes", factor que aunado a su emplazamiento tendiente a la frontera ha mermado en el grado de identificación de los habitantes. Estas ciudades, derivadas de diversos intereses tales como la expansión territorial, la explotación de la tierra o una plataforma para proyectos innovadores, dieron como resultado un urbanismo en el norte del país que rompió con el esquema virreinal, con un carácter totalmente funcional que derivó de un interés capitalista, mismo que ubicó los proyectos con base en las líneas del ferrocarril, los puntos mineros y las haciendas en el país.

\section{La trascendencia se puede notar el crecimiento urbano posterior, \\ TANTO EN LA TRAZA URBANA, LAS DIMENSIONES DE LAS CALLES, LA ARQUITECTURA TANTO EN PERMANENCIA CONSTRUCTIVA COMO LA PERMANENCIA DE ELEMENTOS ARQUITECTÓNICOS.}

En su urbanismo se carece de elementos estructuradores del espacio, se relega en su diseño a los dos elementos simbólicos de la época (la iglesia y el gobierno) y se tiende a trazar una retícula orientada hacia el norte magnético que introduce mayores dimensiones con manzanas de $100 \mathrm{x}$ 
$100 \mathrm{~m}$ y calles de $25 \mathrm{~m}$ de arroyo que incorporan fundamentos en su apropiación como la nomenclatura de las calles.

En la trascendencia de los proyectos favoreció en gran medida el hecho de que existiera un asentamiento previo para impulsar el proyecto urbano y arquitectónico. Se apostó por el desarrollo de puntos estratégicos confluyó en la acumulación de recursos productivos y financieros en una pequeña parte de la población. La concreción de los proyectos responde fundamentalmente a la capacidad de apropiación del proyecto, la congruencia entre la ciudad natal y la de destino, así como la congruencia de las intenciones entre los actores del fenómeno.

La arquitectura es importada con adecuaciones al medio, esta responde al hecho de que cuando el proyecto se genera previo a la llegada de los habitantes se apega a los referentes conocidos y cuando se lleva a cabo posterior a la llegada de los habitantes se apega a las costumbres y materiales locales. La trascendencia se puede notar el crecimiento urbano posterior, tanto en la traza urbana, las dimensiones de las calles, la arquitectura tanto en permanencia constructiva como la permanencia de elementos arquitectónicos.

Si bien en primera instancia se planteó la hipótesis de que su concreción fue determinada desde el diseño y la proyección de los asentamientos (carácter físico), se concluye que fue el aspecto social el determinante para la concreción durante su etapa de ejecución y consolidación. Las características físicas tendientes a un carácter económico dirigido a la racionalización del espacio coadyuvaron en el desarrollo de las poblaciones y previsión del futuro crecimiento, a costa de la homogenización de los desarrollos y la pérdida de los valores simbólicos heredados en el virreinato. Las características sociales, como las intenciones de los desarrolladores (económicas/sociales), la congruencia de las expectativas de los habitantes con la realidad que les esperó y el interés del gobierno mexicano determinaron el funcionamiento del espacio y la apropiación de sus habitantes, fueron la clave que marcó el crecimiento urbano y demográfico de los desarrollos, su concreción a largo plazo y la introducción de un nuevo modelo en el urbanismo de México en el siglo XIX.

\section{FUENTES DE CONSULTA}

Acuña Vigil, P. (2005), Análisis formal del espacio urbano. Aspectos teóricos. Instituto de Investigación de la Facultad de Arquitectura Urbanismo y Artes, Lima.

Aguilar, C. (2015), La historiografía como referencia para el diseño arquitectónico contemporáneo. Caso de Estudio Ensenada Baja California, UABC, Ensenada.

Alvarado, E. (1963), El liberalismo. Imprenta Nacional, San José, Costa Rica.

Calderón, C. (2001), Un siglo de arquitectura en Ensenada, UABC, Ensenada.

De-Vos, J. (1995), Una legislación de grandes consecuencias, COLMEX, México.

González-Milea, A. (2016), "El patrimonio urbano moderno", Región y sociedad, núm. 67, México. 
Lastras, J. (1974), Topolobampo. Albert Kimsey Owen, Los Mochis, El debate. Ledesma, J. (2003), Las comunidades rurales en México durante el siglo XIX, UNAM, México.

Macías, M. (2015), La propiedad agraria durante la época porfiriana, UNAM, México.

Martínez, M. (2009), El proyecto colonizador de México a finales del siglo XIX. Perspectivas comparativas en Latinoamérica, RMCPYS, México.

Schulz, N. (2008), Intenciones en arquitectura, Gili, Barcelona.

Stagno, B. (1997), Arquitectura para una latitud, MENHIR, México. 